\title{
On Computing the Friction Forces Associated with Three-Fingered Grasp
}

\author{
Dominique P. Chevallier*, Shahram Payandeh ${ }^{\star}$ \\ *Centre d'Enseignement et de Recherche en * Experimental Robotics Laboratory(ERL) \\ Mathématiques Appliquées \\ U.R.A - C.N.R.S 1502 . Central 2 \\ La Courtine 93167 Noisy-le Grand Cedex \\ FRANCE \\ School of Engineering Science \\ Burnaby, British Columbia \\ V5A-1S6 \\ CANADA
}

\begin{abstract}
Computation of friction forces between fingers and object for a given external force is important in grasp planning. Based on the required friction forces, the magnitudes of the grasping forces can be adjusted in order to avoid slippage of the object between fingers. A new method is presented for computing the friction forces between the fingertips of a dexterous mechanical hand and the object. Finger-tips are modelled so they are able to exert normal forces and are making point contact with friction with the object. The method of this paper utilizes the geometrical information on the grasp configuration, screw geometry and biorthogonal system. Unlike the method proposed by (Holzmann and McCarthy 85), the method of this paper does not involve matrix inversion and the assumptions under which the computations are valid have a simple geometrical meaning. The method is demonstrated through detailed solutions of two examples.
\end{abstract}

\section{Introduction}

When grasping objects with a dexterous mechanical end-effector, the contact between the fingers of the hand and the object must satisfy a number of conditions (Mason and Salisbury 85), (Kerr and Roth 86), (Li and Sastry 88) and (Payandeh and Goldenberg 88). These range from the force-closure to stability conditions such as, ensuring that the grasped object does not slip between the fingers.

This paper concerns with development of a method for computing the friction forces between the finger-tips and the object when an external force is acting on it. This is an important consideration in the planning aspect of the grasp. For example, when it is planned for the grasped object to make contact with its environment, the expected contact force can be used to determine the required friction forces which contribute in the counterbalancing the external force and hence, the required normal grasping forces.

The paper is organized as follows: section (2) presents some preliminary definitions and assumptions regard to the statement of our objective; section (3) presents the main results of this paper; section (4) presents the detailed solutions of two examples and section (5) presents concluding remarks. 


\section{Preliminary}

This section presents some definitions and assumptions in regard to the objective of this paper and defines the statement of the problem.

Assumption 1 The grasp configuration consists of three fingers where only the finger-tips are making contact with the object.

Assumption 2 Finger-tips are made of solid semi-spherical material which can make point contact with friction with the object.

Definition 1 The three fingered grasp is defined by:

- three points $p_{1}, p_{2}$ and $p_{3}$ (contact points),

- the normalized screws $\nu_{1}, \nu_{2}$ and $\nu_{3}$, where for $i=1,2,3$ we have: (Appendix)

$$
\begin{aligned}
& {\left[\nu_{i} \mid \Omega \nu_{i}\right]=1} \\
& p_{i} \in \text { axis of } \nu_{i}
\end{aligned}
$$

The screw $\nu_{i}$ describes the oriented normal to the surface of the grasped object at the point $p_{i}$. The normal component of the grasping force which acts on the object at point $p_{i}$ is described by $N \nu_{i}$, where $N$ is a real number $(N \in \mathbf{R})$ representing the magnitude of the normal force.

Let $\Pi_{i}$ denote the tangential plane to the object at $p_{i}$. We then define the following:

Definition 2 Friction force is defined by a wrench $F_{i}$ with $p_{i} \in\left(\right.$ axis of $\left.F_{i}\right)$ and axis $F_{i} \subset \Pi_{i}$, that is:

- $F_{i} \in \mathcal{D}$ (Appendix)

- $F_{i}\left(p_{i}\right)=0 \quad$ (The moment of $F_{i}$ about $p_{i}$ is equal to zero)

- $\left[F_{i} \mid \Omega \nu_{i}\right]=0$ (i.e. orthogonality of the axis $F_{i}$ and $\nu_{i}$ )

The general statement of the objective can be described as follows:

Given,

1. the points $p_{1}, p_{2}$ and $p_{3}$;

2. the screw $\nu_{1}, \nu_{2}$ and $\nu_{3}$ and the corresponding magnitudes $\left(N_{1}, N_{2}, N_{3}\right)$ and,

3. the external wrench $\Phi$ which acts on the object; 
Find $\left(F_{1}, F_{2}, F_{3}\right)$ such that:

$$
\left\{\begin{array}{l}
\Phi+\sum_{i=1}^{3} N_{i} \nu_{i}+\sum_{i=1}^{3} F_{i}=0 \\
F_{i} \text { satisfies conditions of definition }(2) \text { for } \mathrm{i}=1,2,3
\end{array}\right.
$$

Specifically, the objective of this paper is stated in the following. Let $\mathcal{Z}_{i}$ be the vector subspace of $\mathcal{D}$ which is defined as:

$$
\mathcal{Z}_{i}=\left\{\mathbf{X} \in \mathcal{D} \mid \mathbf{X}\left(p_{i}\right)=0\right\}
$$

and let $\mathcal{F}_{i}$ be the vector subspace of $\mathcal{Z}_{i}$ defined by:

$$
\mathcal{F}_{i}=\left\{\mathbf{X} \in \mathcal{D} \mid \mathbf{X}\left(p_{i}\right)=0,\left[\mathbf{X} \mid \Omega \nu_{i}\right]=0\right\} \quad \nu_{i} \in \mathcal{Z}_{i}
$$

Based on the above, a force acting from the finger on the object at $p_{i}$ is described by a wrench belonging to $\mathcal{Z}_{i}$. Whenever it is a normal force this wrench takes the form $N \nu_{i}$ and whenever it is a tangential force it is described by a wrench of $F_{i}$.

The problem of computing the friction force associated with the three fingered grasp can be stated as the answers to the following questions:

1) Does the relation $\mathcal{F}_{1} \oplus \mathcal{F}_{2} \oplus \mathcal{F}_{3}=\mathcal{D}$ hold? (In other words, does every member of $\mathcal{D}$ splits into the sum of three uniquely defined members of $\mathcal{F}_{1}, \mathcal{F}_{2}$ and $\mathcal{F}_{3}$ ?)

2) What are the analytical expressions of the projections:

$$
\mathcal{D} \rightarrow \mathcal{F}_{i} \quad i=1,2,3
$$

\section{Main Results}

This section presents the main theorem which are used to show the answer to the first and second questions and the analytical expressions for obtaining the projections $\mathcal{D} \rightarrow \mathcal{F}_{i}$.

Theorem 1 Suppose that the planes $\Pi_{1}, \Pi_{2}$ and $\Pi_{3}$ intersect at a single point $p$ ( $p$ is at infinity whenever the axis $\nu_{1}, \nu_{2}$ and $\nu_{3}$ are parallel to a same plane or whenever two planes are parallel). Then the following properties are equivalent:

a) the points $p, p_{1}, p_{2}, p_{3}$ do not belong to the same plane,

b) $\mathcal{D}=\mathcal{F}_{1} \oplus \mathcal{F}_{2} \oplus \mathcal{F}_{3}$

In the sequel, when the three letters $i, j, k$ appear in a formula, it means that $(i, j, k)$ is a circular permutation of $(1,2,3)$. Let $\mathcal{S}_{i}$ and $\Sigma_{i}(i=1,2,3)$ be normalized screws such that:

$$
\left\{\begin{array}{l}
\left(\text { axis of } \mathcal{S}_{i}\right)=\Pi_{j} \cap \Pi_{k}, \text { if } \Pi_{j} \text { and } \Pi_{k} \text { are not parallel } \\
\mathcal{S}_{i}=\Omega \nu_{j}, \text { if } \Pi_{j} \text { and } \Pi_{k} \text { are parallel } \\
\Sigma_{i}\left(p_{j}\right)=\Sigma_{i}\left(p_{k}\right)=0
\end{array}\right.
$$

When $\Pi_{j}$ and $\Pi_{k}$ are parallel, $\Omega \nu_{j}= \pm \Omega \nu_{k}$ and the choice $\mathcal{S}_{i}=\Omega \nu_{k}$ would be equivalent. The axis of $\Sigma_{i}$ is the line $p_{j} p_{k}$.

Let us define a vector subspace of $\mathcal{D}$ by $\mathcal{F}_{(i j)}=\mathcal{F}_{i}+\mathcal{F}_{j}$ (i.e. the set of sums of members of $\mathcal{F}_{i}$ and of $\mathcal{F}_{j}$ ).

We first prove two lemmas. 
Lemma 1 Suppose that $p_{i}$ and $p_{j}$ are not in $\Pi_{i} \cap \Pi_{j}$, then:

i) For $i \neq j, \mathcal{F}_{(i j)}$ is a four dimensional vector subspace of $\mathcal{D}$

ii) The reciprocal vector subspace of $\mathcal{F}_{(i j)}$ is

$$
\mathcal{F}_{(i j)}^{\perp}=\operatorname{Space}\left\{\mathcal{S}_{k}, \Sigma_{k}\right\}
$$

The reciprocal $\mathcal{F}^{\perp}$ of a subspace $\mathcal{F}$ of $\mathcal{D}$ means the orthogonal subspace with respect to the inner product $[\cdot \mid \cdot]$ :

$$
\mathcal{F}^{\perp}=\{\mathbf{Y} \in \mathcal{D} \mid[\mathbf{X} \mid \mathbf{Y}]=0 \quad \text { for all } \mathbf{X} \text { in } \mathcal{F}\}
$$

Proof. Since $\nu_{i} \in \mathcal{Z}_{i}$ and $\left[\nu_{i} \mid \Omega \nu_{i}\right]=1$, the linear form $\mathbf{X} \mapsto\left[\mathbf{X} \mid \Omega \nu_{i}\right]$ is not zero for all $\mathbf{X} \in \mathcal{Z}_{i}$ and its kernel $\mathcal{F}_{i}$ is therefore a two dimensional subspace of the three dimensional space $\mathcal{Z}_{i}$. Now we prove that

$$
\mathcal{F}_{i} \cap \mathcal{F}_{j}=\{0\} \quad, \quad(i \neq j)
$$

Under the hypothesis of the lemma, $p_{i} \neq p_{j}$ and $\mathcal{Z}_{i} \cap \mathcal{Z}_{j}$ is the one dimensional vector subspace of $\mathcal{D}$ spanned by $\Sigma_{k}$. Obviously: $\mathcal{F}_{i} \cap \mathcal{F}_{j} \subset \mathcal{Z}_{i} \cap \mathcal{Z}_{j}$. The axis of a non-zero element of $\mathcal{F}_{i} \cap \mathcal{F}_{j}$ would be the line $p_{i} p_{j}$ (the axis of $\Sigma_{k}$ ) and would be included in $\Pi_{i} \cap \Pi_{j}$. Therefore such a screw may not exist and (6) is proved. Property i) follows from (6) and the dimension of $\mathcal{F}_{i}$ and $\mathcal{F}_{j}$.

The screws $\mathcal{S}_{k}$ and $\Sigma_{k}$ are linearly independent (since the axis of $\mathcal{S}_{k}$, if it exists, can not be equal to the axis of $\Sigma_{k}$ ) and belong to $\mathcal{F}_{(i j)}^{\perp}$ (In fact $\Sigma_{k}$ is reciprocal to all the screws belonging to $\mathcal{Z}_{i}+\mathcal{Z}_{j}$, a space containing $\left.\mathcal{F}_{(i j)}\right)$. If $\Pi_{i}$ and $\Pi_{j}$ are not parallel, the axis of $\mathcal{S}_{k}$ meets or is parallel to the axis of all the screws belonging to $\mathcal{F}_{i}$ or to $\mathcal{F}_{j}$, hence $\mathcal{S}_{k}$ is reciprocal to all these screws and to the screws belonging to $\mathcal{F}_{(i j)}$. The previous property remains true if $\Pi_{i}$ and $\Pi_{j}$ are parallel and $\mathcal{S}_{k}=\Omega \nu_{i}$.

Since $\mathcal{F}_{(i j)}^{\perp}$ is a two dimensional vector subspace, property ii) follows from these remarks. $\square$

Lemma 2 Under the assumptions of the previous lemma, the following properties are equivalent:

a) The lines $p_{i} p_{j}$ and $p p_{k}$ are not in a same plane,

B) $\mathcal{F}_{(i j)} \cap \mathcal{F}_{k}=\{0\}$

Proof. From lemma 1 we have:

$$
\mathbf{X} \in \mathcal{F}_{(i j)} \cap \mathcal{F}_{k} \Longleftrightarrow \mathbf{X} \in \mathcal{F}_{k} \text { and }\left[\mathbf{X} \mid \mathcal{S}_{k}\right]=0 \text { and }\left[\mathbf{X} \mid \Sigma_{k}\right]=0
$$

First we prove that $(\alpha)$ implies $(\beta)$. The axis of a non-zero element $\mathbf{X}$ of $\mathcal{F}_{k}$ such that $\left[\mathbf{X} \mid \mathcal{S}_{k}\right]=$ 0 must be a line through $p_{k}$ lying in $\Pi_{k}$ and in a plane containing the line $\Pi_{i} \cap \Pi_{j}$; therefore it must be the line $p p_{k}$ (if $\Pi_{i}$ and $\Pi_{j}$ are parallel, $p p_{k}$ is the line through $p_{k}$ and which is parallel to these planes). If moreover $\left[\mathbf{X} \mid \Sigma_{k}\right]=0$, this line must also be in a plane containing the line $p_{i} p_{j}$, a contradiction. Hence no non-zero element belongs to $\mathcal{F}_{(i j)} \cap \mathcal{F}_{k}$.

Now we prove that $(\beta)$ implies $(\alpha)$ by proving that not $(\alpha) \operatorname{implies} \operatorname{not}(\beta)$. If the lines $p_{i} p_{j}$ and $p p_{k}$ would be in a same plane for any non-zero element of $\mathcal{F}_{k}$ the axis of which is the line $p p_{k}$ would be in $\left\{\mathcal{S}_{k}, \Sigma_{k}\right\}^{\perp}=\mathcal{F}_{(i j)}$ and also in $\mathcal{F}_{(i j)} \cap \mathcal{F}_{k}$ ( a contradiction). 
Proof of Theorem 1. Assuming the first property, the hypothesis of lemma 1 and 2 and property $(\alpha)$ hold for all choices of $(i, j, k)$, hence condition $(\beta)$ also holds and this prove the second property.

Conversely, assuming the second property, the property $(\beta)$ holds for all choices of $(i, j, k)$ and also $(\alpha)$ from lemma 2. This prove the first property.

For finding the analytical expression of the projection $\mathcal{P}_{i}: \mathcal{D} \rightarrow \mathcal{F}_{i}$ we first build a remarkable basis of $\mathcal{F}_{i}$ for $(i=1,2,3)$.

Let $u_{i}$ be normalized vectors along the line $p p_{i}$; whenever $p$ is at infinity we may choose $u_{1}=u_{2}=u_{3}=u$. Let $\xi_{i}$ be the screws such that:

$$
\xi_{i} \in \mathcal{Z}_{i}, \quad \omega_{\xi_{i}}=u_{i}, \quad i=1,2,3 .
$$

Then,

$$
\xi_{i} \in \mathcal{F}_{i}, \quad i=1,2,3 .
$$

The screws $\Sigma_{1}, \Sigma_{2}$ and $\Sigma_{3}$ generate a three-system of screws $\mathcal{T}$, that is the set of screws:

$$
\alpha_{1} \Sigma_{1}+\alpha_{2} \Sigma_{2}+\alpha_{3} \Sigma_{3} \text { with } \alpha_{1}, \alpha_{2}, \alpha_{3} \in \mathbf{R}
$$

which is also the set of screws with zero pitch and with axis lying in the plane $p_{1}, p_{2}, p_{3}$ (these screws describe the forces acting along lines lying in this plane).

For all $i$, it is possible to choose a screw $\eta_{i}$ such that:

$$
\eta_{i} \in \mathcal{F}_{i} \cap \mathcal{T} \text {, and } \eta_{i} \neq 0
$$

A screw $\eta$ like it was defined in equation $(7)$ is in $\mathcal{Z}_{i}$ if $\alpha_{i}=0$, that is if:

$$
\eta=\alpha_{j} \Sigma_{j}+\alpha_{k} \Sigma_{k}, \quad \alpha_{j}, \alpha_{k} \in \mathbf{R}
$$

In order for the previous screw $\eta$ to be also in $\mathcal{F}_{i}$ it is necessary that,

$$
\left[\Omega \nu_{i} \mid \eta\right]=\left[\Omega \nu_{i} \mid \alpha_{j} \Sigma_{j}+\alpha_{k} \Sigma_{k}\right]=\alpha_{j}\left[\Omega \nu_{i} \mid \Sigma_{j}\right]+\alpha_{k}\left[\Omega \nu_{i} \mid \Sigma_{k}\right]=0
$$

A solution is:

$$
\alpha_{j}=\left[\Omega \nu_{i} \mid \Sigma_{k}\right], \quad \alpha_{k}=-\left[\Omega \nu_{i} \mid \Sigma_{j}\right]
$$

and thus,

$$
\eta_{i}=\left[\Omega \nu_{i} \mid \Sigma_{k}\right] \Sigma_{j}-\left[\Omega \nu_{i} \mid \Sigma_{j}\right] \Sigma_{k}
$$

Under the first assumption of theorems, for each $i, \xi_{i}$ and $\eta_{i}$ are linearly independent screws (the axis of $\eta_{i}$ lies in the plane $p_{1} p_{2} p_{3}$ and the axis of $\xi_{i}$, that is the line $p_{i} p$, does not also lie in this plane). In other words:

$$
\left\{\xi_{i}, \eta_{i}\right\} \quad \text { is a basis of } \mathcal{F}_{i}
$$

Let $\mathbf{X} \in \mathcal{D}$ be a wrench which acts on the grasped object. This wrench can be expanded as:

$$
\mathbf{X}=\sum_{k=1}^{3} x_{k} \xi_{k}+y_{k} \eta_{k}, \quad x_{k}, y_{k} \in \mathbf{R}
$$


then,

$$
\begin{aligned}
{\left[\mathbf{X} \mid \mathcal{S}_{i}\right] } & =y_{i}\left[\eta_{i} \mid \mathcal{S}_{i}\right] \\
{\left[\mathbf{X} \mid \Sigma_{i}\right] } & =x_{i}\left[\xi_{i} \mid \Sigma_{i}\right]
\end{aligned}
$$

The assumption $\left(p_{1}, p_{2}, p_{3}, p\right.$ are not in the same plane) implies that $\left[\xi_{i} \mid \Sigma_{i}\right] \neq 0$ and $\left[\eta_{i} \mid \mathcal{S}_{i}\right] \neq 0$ and the solution is:

$$
\left\{\begin{array}{l}
x_{i}=\frac{1}{\left[\xi_{i} \mid \Sigma_{i}\right]}\left[\mathbf{X} \mid \Sigma_{i}\right] \\
y_{i}=\frac{1}{\left[\eta_{i} \mid \mathcal{S}_{i}\right]}\left[\mathbf{X} \mid \mathcal{S}_{i}\right]
\end{array}\right.
$$

The projection $\mathcal{P}_{i}$ can then be written as:

$$
P_{i}=\frac{1}{\left[\xi_{i} \mid \Sigma_{i}\right]} \Sigma_{i} \otimes \xi_{i}+\frac{1}{\left[\eta_{i} \mid \mathcal{S}_{i}\right]} \mathcal{S}_{i} \otimes \eta_{i}, \quad i=1,2,3
$$

where, for example, $\Sigma_{i} \otimes \xi_{i}$ means the linear operator $\mathbf{X}: \mapsto\left[\mathbf{X} \mid \Sigma_{i}\right] \xi_{i}$.

Remark 1 The two bases $\left(\mathcal{S}_{1}, \mathcal{S}_{2}, \mathcal{S}_{3}, \Sigma_{1}, \Sigma_{2}, \Sigma_{3}\right)$ and $\left(\xi_{1}, \xi_{2}, \xi_{3}, \eta_{1}, \eta_{2}, \eta_{3}\right)$ make a biorthogonal system in the vector space $\mathcal{D}$ endowed with the inner product $[\cdot \mid \cdot]$, that is all screw of one system is orthogonal to all, but one screw of the other.

\section{Examples}

Example 1: A polyhedral object is grasped by three finger having stiff finger-tip (see Figure 1). The points of contact with respect to the object's reference coordinate frame is given as:

$$
\begin{aligned}
& p_{1}=(0.0,2.0,5.0) \\
& p_{2}=(2.0,0.0,4.0) \\
& p_{3}=(2.0,2.0,0.0)
\end{aligned}
$$

The normalized screws representing the contact normals $\left(n_{1}, n_{2}, n_{3}\right)$ at the three points with respect to o are:

$$
\begin{aligned}
& \nu_{1}=(1,0,0 ; 0,5,-2) \\
& \nu_{2}=(0,1,0 ;-4,0,2) \\
& \nu_{3}=(0,0,1 ; 2,-2,0)
\end{aligned}
$$

Given the grasp configuration, we can compute:

$$
\begin{aligned}
& u_{1}=(0.0,0.371,0.928) \\
& u_{2}=(0.447,0.0,0.894) \\
& u_{3}=(0.707,0.707,0.0)
\end{aligned}
$$

The normalized screws $\xi_{1}, \xi_{2}$ and $\xi_{3}$ can be determined as: (see Figure 2)

$$
\begin{aligned}
& \xi_{1}=(0.0,0.371,0.928 ; 0.0,0.0,0.0) \\
& \xi_{2}=(0.447,0.0,0.894 ; 0.0,0.0,0.0) \\
& \xi_{3}=(0.707,0.707,0.0 ; 0.0,0.0,0.0)
\end{aligned}
$$




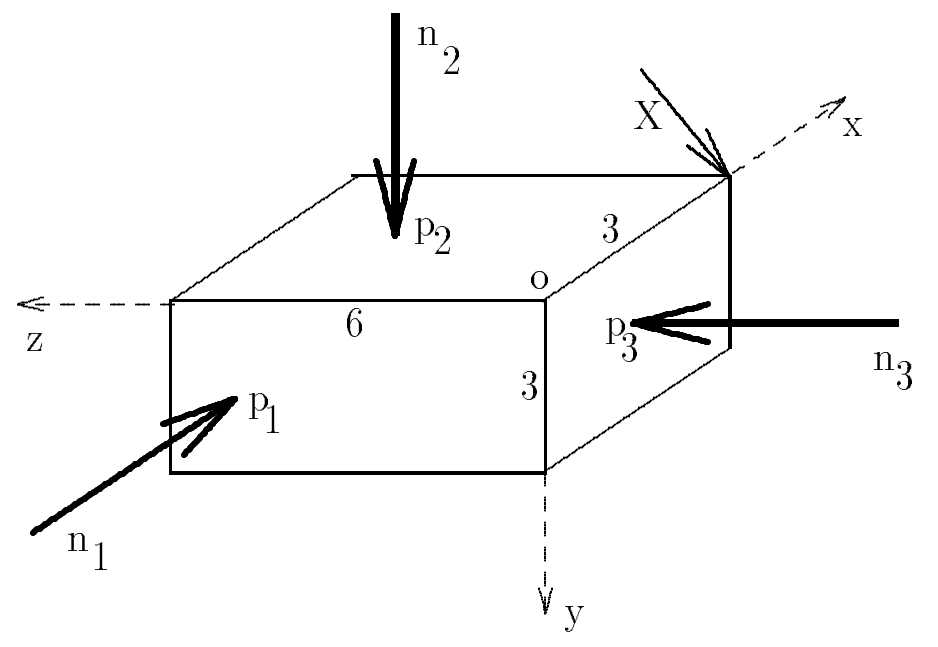

Figure 1: A three fingered grasp

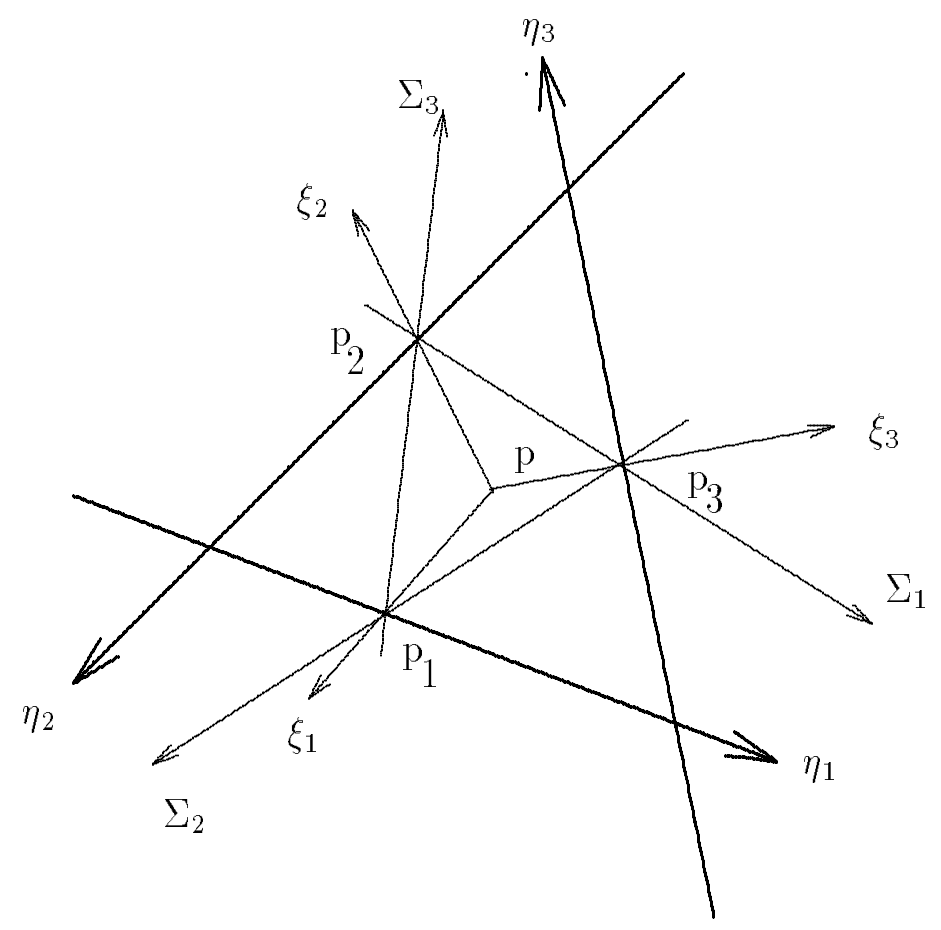

Figure 2: Definition of screw $\xi_{i}$ 


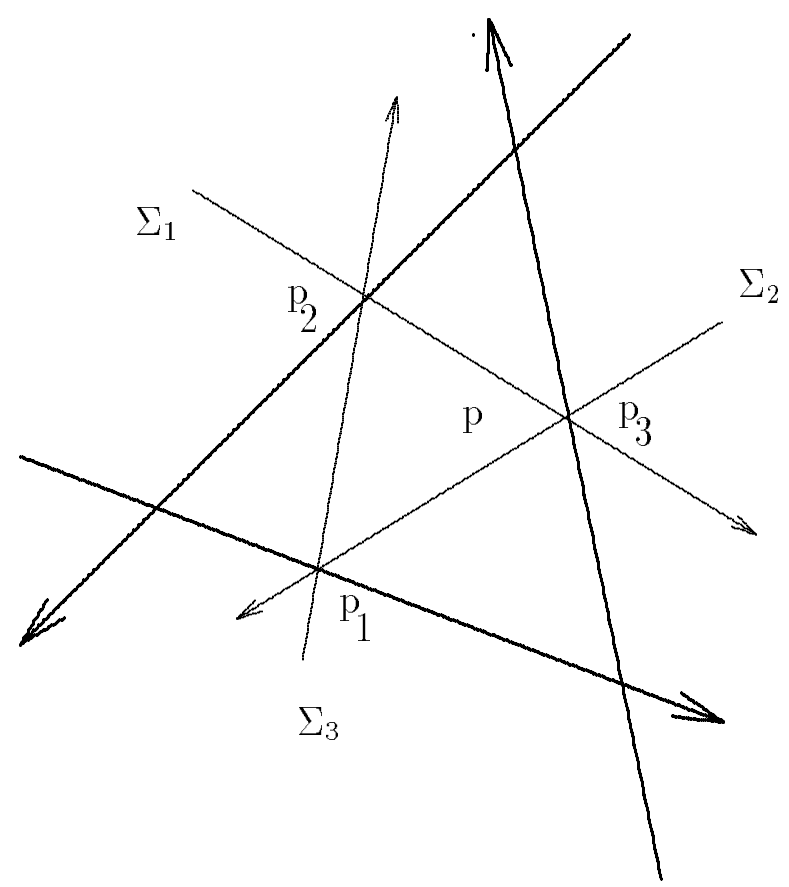

Figure 3: Definition of screw $\Sigma_{i}$

The screws $\Sigma_{1}, \Sigma_{2}$ and $\Sigma_{3}$ which are normalized screws through points $\left(p_{1}, p_{2}\right),\left(p_{2}, p_{3}\right)$ and $\left(p_{3}, p_{1}\right)$ respectively are defined using the following general Plücker line coordinates representation, i.e. for two point $p_{i}=\left(x_{i}, y_{i}, z_{i}\right)$ and $p_{j}=\left(x_{j}, y_{j}, z_{j}\right)$ : (screw with zero pitch)

$$
\begin{aligned}
& L=x_{j}-x_{i} \\
& M=y_{j}-y_{i} \\
& N=z_{j}-z_{i} \\
& P=y_{i} N-z_{i} M \\
& Q=z_{i} L-x_{i} N \\
& R=x_{i} M-y_{i} L
\end{aligned}
$$

Therefore, the normalized screw $\Sigma_{1}, \Sigma_{2}$ and $\Sigma_{3}$ are determined to be: (see Figure 3 )

$$
\begin{aligned}
& \Sigma_{1}=(0.0,0.447,-0.894 ;-1.788,1.788,0.894) \\
& \Sigma_{2}=(-0.371,0.0,0.982 ; 1.856,-1.856,0.742) \\
& \Sigma_{3}=(0.666,-0.666,-0.333 ; 2.664,3.33,-1.332)
\end{aligned}
$$

Following the definition of inner product in $\mathcal{D}$ (see Appendix), the screws $\eta_{1}, \eta_{2}$ and $\eta_{3}$ can 
be find from equation (8) as:

$$
\begin{aligned}
\eta_{1}= & 0.666(-0.371,0.0,0.982 ; 1.856,-1.856,0.742) \\
& +0.371(0.666,-0.666,-0.333 ; 2.664,3.33,-1.332) \\
= & (0.0,-0.247,0.53 ; 2.224,0.0,0.0) \\
\eta_{2}= & 0.447(0.666,-0.666,-0.333 ; 2.664,3.33,-1.332) \\
& +0.666(0.0,0.447,-0.894 ;-1.788,1.788,0.894) \\
= & (0.297,0.0,0.744 ; 0.0,2.629,0.0) \\
\eta_{3}= & 0.982(0.0,0.447,-0.894 ;-1.788,1.788,0.894) \\
& +0.894(-0.371,0.0,0.982 ; 1.856,-1.856,0.742) \\
= & (-0.331,0.438,0.0 ; 0.0,0.0,1.541)
\end{aligned}
$$

The normalized screws $\mathcal{S}_{1}, \mathcal{S}_{2}$ and $\mathcal{S}_{4}$ can be determined from the geometry of the grasp configuration as:

$$
\begin{aligned}
& \mathcal{S}_{1}=\Pi_{2} \cap \Pi_{3}=(1,0,0 ; 0,0,0) \\
& \mathcal{S}_{2}=\Pi_{3} \cap \Pi_{1}=(0,1,0 ; 0,0,0) \\
& \mathcal{S}_{3}=\Pi_{1} \cap \Pi_{2}=(0,0,1 ; 0,0,0)
\end{aligned}
$$

Let a wrench with the intensity of $10 \mathrm{lbf}$ be acting on the object about the normalized screw defined by, (see Figure 1)

$$
\begin{aligned}
\mathbf{X}= & 10(-0.707,0.0,-0.707 ; 0.0,2.12,0.0) \\
& (-7.07,0.0,-7.07 ; 0.0,21.2,0.0)
\end{aligned}
$$

This wrench can split into:

$$
\mathbf{X}=x_{1} \xi_{1}+y_{1} \eta_{1}+x_{2} \xi_{2}+y_{2} \eta_{2}+x_{3} \xi_{3}+y_{3} \eta_{3}
$$

where we can compute from equation (10) the coefficients $x_{i}$ and $y_{i}$, as:

$$
\begin{array}{cc}
x_{1}=\frac{1}{\left[\xi_{1} \mid \Sigma_{1}\right]}\left[\mathbf{X} \mid \Sigma_{1}\right]=10.6 & y_{1}=\frac{1}{\left[\eta_{1} \mid \mathcal{S}_{1}\right]}\left[\mathbf{X} \mid \mathcal{S}_{1}\right]=0 ., \\
x_{2}=\frac{1}{\left[\xi_{2} \mid \Sigma_{2}\right]}\left[\mathbf{X} \mid \Sigma_{2}\right]=-12.3 & y_{2}=\frac{1}{\left[\eta_{2} \mid \mathcal{S}_{2}\right]}\left[\mathbf{X} \mid \mathcal{S}_{2}\right]=-8.09, \\
x_{3}=\frac{1}{\left[\xi_{3} \mid \Sigma_{3}\right]}\left[\mathbf{X} \mid \Sigma_{3}\right]=-5.55 & y_{3}=\frac{1}{\left[\eta_{3} \mid \mathcal{S}_{3}\right]}\left[\mathbf{X} \mid \mathcal{S}_{3}\right]=0 . .
\end{array}
$$

The friction wrenches are then calculated to be:

$$
\begin{aligned}
& F_{1}=(0.0,-3.71,9.28 ; 0.0,0.0,0.0) \\
& F_{2}=(-7.9,0.0,-17.07 ; 0.0,-21.2,0.0) \\
& F_{3}=(-3.92,-3.92,0.0 ; 0.0,0.0,0.0)
\end{aligned}
$$

Example 2: A cylindrical object of radius 1 is grasped as is shown in the Figure (4). The contact points are given as: $p_{1}=(1,0,0), p_{2}=(-0.5,0.866,0$. $)$ and $p_{3}=(-0.5,-0.866,0$. $)$. The normalized screws representing the contact normals $\left(n_{1}, n_{2}, n_{3}\right)$ are written as: 


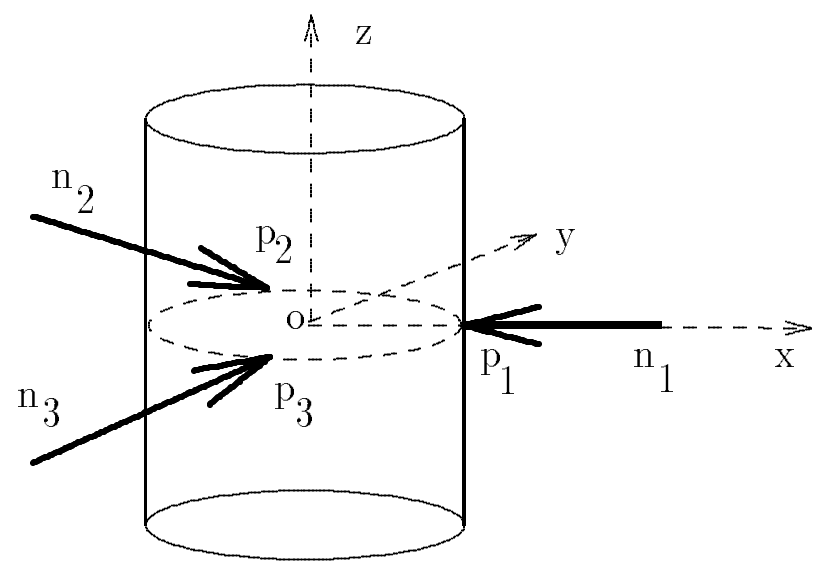

Figure 4: The grasp configuration of example (2)

$$
\begin{aligned}
& \nu_{1}=(-1,0,0 ; 0,0,0) \\
& \nu_{2}=(0.5,-0.866,0.0 ; 0,0,0) \\
& \nu_{3}=(0.5,0.866,0.0 ; 0,0,0)
\end{aligned}
$$

From the definition, for the case when $\nu_{1}, \nu_{2}$ and $\nu_{3}$ are parallel to the same plane, we choose $u_{1}=u_{2}=u_{3}=u=(0,0,1)$. As a result the normalized screws $\xi_{1}, \xi_{2}$ and $\xi_{3}$ can be computed to be:

$$
\begin{aligned}
& \xi_{1}=(0,0,1 ; 0,-1,0) \\
& \xi_{2}=(0,0,1 ; 0.866,0.5,0) \\
& \xi_{3}=(0,0,1 ;-0.866,0.5,0)
\end{aligned}
$$

From the grasp configuration, the normalized screws $\Sigma_{i}$ and $\mathcal{S}_{i}$ can be computed to be:

$$
\begin{aligned}
& \Sigma_{1}=(0,-1,0 ; 0,0,0.5) \\
& \Sigma_{2}=(0.866,0.5,0 ; 0,0,0.5) \\
& \Sigma_{3}=(-0.866,0.5,0 ; 0,0,0.5)
\end{aligned}
$$

and,

$$
\begin{aligned}
& \mathcal{S}_{1}=(0,0,1 ; 0,2,0) \\
& \mathcal{S}_{2}=(0,0,1 ;-1.732,-1,0) \\
& \mathcal{S}_{3}=(0,0,1 ; 1.732,-1,0)
\end{aligned}
$$

From equation (8), we have:

$$
\begin{aligned}
& \eta_{1}=(0,0.866,0 ; 0,0,-0.433) \\
& \eta_{2}=(-0.749,-0.433,0 ; 0,0,-0.749) \\
& \eta_{3}=(0.749,-0.433,0 ; 0,0,-0.433)
\end{aligned}
$$

Let the only force which can act on the grasped object be the object's own weight of 10(lbf) acting at its center of gravity located at point o or, $\mathbf{X}=(0,0,-10,0,0,0)$. From equation (10), we have : $\left[\mathbf{X} \mid \Sigma_{i}\right]=-5$ for $i=1,2,3$. Also, for the grasp configuration of this example we have: $\left[\mathbf{X} \mid \mathcal{S}_{i}\right]=0$ for $i=1,2,3$. The product $\left[\xi_{i} \mid \Sigma_{i}\right]=1.5$ for $i=1,2,3$. 
Therefore, the friction forces associated with the three finger grasp configuration are computed to be:

$$
\begin{aligned}
& F_{1}=x_{1} \xi_{1}=(0,0,-3.33 ; 0,3.33,0) \\
& F_{2}=x_{2} \xi_{2}=(0,0,-3.33 ;-2.883,-1.665,0) \\
& F_{3}=x_{3} \xi_{3}=(0,0,-3.33 ; 2.883,-1.665,0)
\end{aligned}
$$

\section{Conclusion}

This paper outlined a new method for computing the friction forces associated with three fingered grasp. Given the geometry of the grasped object, the locations of contact points and the surface normals, we are able to determine the magnitude of the friction forces as a function of the external applied force on the object.

Computation of friction forces as function of the external forces plays an important role in grasp planning strategies for determining the magnitude of the grasping forces. This is based on the assumption that at each contact point between the fingers and the object there exist a direct relationship between friction and the normal force (i.e. $F_{i}=\mu N \nu_{i}$ where $\mu$ is the coefficient of static friction). Hence by determining $F_{i}$ the magnitude of the grasping forces can be obtained.

The method of this paper requires:

- building of the screws $\mathcal{S}_{i}, \Sigma_{i}$ and $\xi_{i}$ and $\eta_{i}$ which are available from the grasp configuration data,

- the computation of some inner products $[\cdot \mid \cdot]$.

The results of this paper is more clear and does not required any matrix inversion comparing with (Holzmann and McCarthy 85).

\section{References}

Chevallier, D. P. 1991. Lie Algebras, Modules, Dual Quternions and Algebraic Methods in Kinematics. Mechanisms and Machine Theory. Vol. 26(6), pp. 613-627

Holzmann, W. and McCarthy, M. 1985. Computing the Friction Forces Associated with a Three-Fingered Grasp. IEEE Journal of Robotics and Automation. Vol. RA-1(4). pp. 206-210

Kerr, J. and Roth, B. 1986. Analysis of Multi-fingered Hands. The International Journal of Robotics Research. Vol. 4(4). pp. 3-17

Li, Z. and Sastry, S. 1988. Task-Oriented Optimal Grasping by Multi-fingered Robot Hands. IEEE Journal of Robotics and Automation. Vol. 4(1). pp. 32-44

Mason, M. and Salisbury, J. K. 1985. Robot Hand and the Mechanics of Manipulation, The MIT Press

Payandeh, S. and Goldenberg, A. 1988 . Grasp Impedance: Examples of Fingers' Targeted Impedance, Proceedings of 3rd IEEE International Symposium on Intelligent Control. Arlington. 


\section{Appendix}

In this appendix we summarize the definition of the algebraic operations on screws as used in the article. The detail are expounded in (Chevallier 91).

In the following $\varepsilon$ denotes the tridimensional affine Euclidean space, $\mathcal{E}$ the underlying vector space; to every pair $(a, b)$ of points in $\varepsilon$ is associated a vector $\overrightarrow{a b}$ in $\mathcal{E}$ so that Chasles' relation holds.

Definition 3 Vector Space $\mathcal{D}$. It is well known that to every screw is associated a vector space field $\mathbf{X}: \varepsilon \rightarrow \mathcal{E}$ with the property (helicoidal field)

$$
\mathbf{X}(b)=\mathbf{X}(a)+\omega_{\mathbf{X}} \times \overrightarrow{a b} \text { for all } a \text { and } b \text { in } \varepsilon
$$

All these field make a real vector space $\mathcal{D}$ and the map $\mathbf{X} \mapsto \omega_{\mathrm{X}}$ is linear.

Let $W$ be a wrench representation with $W=(f ; g)$ with respect to the origin 0 . Then the associated Helicoidal field is:

$$
\mathbf{W}(p)=g+f \times \overrightarrow{o p}, \quad p \in \varepsilon
$$

(the moment of the wrench about the point $p$ ) and the mapping $\mathbf{X} \mapsto \omega_{\mathbf{X}}$ can be written as: $W \mapsto f$ (force). Let $\mathrm{T}$ be a twist representation with $T=(\omega ; v)$ with respect to the origin $\mathrm{o}$, then the associated helicoidal field $(\mathbf{V}(p)=\mathbf{v}+\omega \times \overrightarrow{o p})$ describes the velocity of a rigid motion of angular velocity $\omega$.

Definition 4 Lie bracket in $\mathcal{D}$. For $\mathbf{X}$ and $\mathbf{Y}$ in $\mathcal{D}$, define the vector field $\mathbf{U}: \varepsilon \rightarrow \mathcal{E}$ through

$$
\mathbf{U}(p)=\omega_{\mathbf{X}} \times \mathbf{Y}(p)-\omega_{\mathbf{Y}} \times \mathbf{X}(p) .
$$

Then it is easy to show that $\mathbf{U} \in \mathcal{D}$ and $\omega_{\mathbf{U}}=\omega_{\mathbf{X}} \times \omega_{\mathbf{Y}}$. This helicoidal field is denoted by $[\mathbf{X}, \mathbf{Y}]$.

Definition 5 Inner Product in $\mathcal{D}$. For $\mathbf{X}$ and $\mathbf{Y}$ in D, define the real number:

$$
[\mathbf{X} \mid \mathbf{Y}]=\omega_{\mathbf{X}} \cdot \mathbf{Y}(p)+\omega_{\mathbf{Y}} \cdot \mathbf{X}(p)
$$

The relation $[\mathbf{X} \mid \mathbf{Y}]=0$ means that the screws associated with $\mathbf{X}$ and $\mathbf{Y}$ are reciprocal.

For a wrench $W$ and a twist $T$ as above, the inner product $[\mathbf{W} \mid \mathbf{T}]$ (of the associated helicoidal field) equal $\mathbf{f} \cdot \mathbf{v}+g \cdot \omega$ the work done by the wrench over the twist.

Definition 6 Operation $\Omega$. For $\mathbf{X}$ in $\mathcal{D}$, define the constant field $\Omega \mathbf{X}$ such that

$$
\Omega \mathbf{X}(p)=\omega_{\mathbf{X}} \quad \text { for all } p \in \varepsilon
$$

Then $\Omega$ is a linear operation in $\mathcal{D}$

For example, with the previous notation, $\Omega W=(0 ; f)$, and $\Omega T=(0 ; \omega)$. (The mapping $\Omega$ is different of the mapping $\mathbf{X} \mapsto \omega_{\mathbf{X}}$ which does not map a screw on a screw). 